\title{
L'univers du pèlerinage des malades à Lourdes (Rurudo shôbyôsha junrei no sekai)
}

Tôkyô, Chisen shokan, 2006, 556 p.

Fabienne Duteil-Ogata et Jean-Pierre Berthon

\section{OpenEdition}

\section{Journals}

Édition électronique

URL : http://journals.openedition.org/assr/16073

DOI : $10.4000 /$ assr. 16073

ISSN : $1777-5825$

Éditeur

Éditions de l'EHESS

Édition imprimée

Date de publication : 1 juin 2008

ISBN : 978-2-7132-2190-3

ISSN : 0335-5985

Référence électronique

Fabienne Duteil-Ogata et Jean-Pierre Berthon, « L'univers du pèlerinage des malades à Lourdes (Rurudo shôbyôsha junrei no sekai) », Archives de sciences sociales des religions [En ligne], 142 | avriljuin 2008, document 142-58, mis en ligne le 10 décembre 2010, consulté le 21 septembre 2020. URL : http://journals.openedition.org/assr/16073 ; DOI : https://doi.org/10.4000/assr.16073

Ce document a été généré automatiquement le 21 septembre 2020.

(C) Archives de sciences sociales des religions 


\section{L'univers du pèlerinage des malades à Lourdes (Rurudo shôbyôsha junrei no sekai)}

Tôkyô, Chisen shokan, 2006, 556 p.

Fabienne Duteil-Ogata et Jean-Pierre Berthon

1 L'univers du pèlerinage des malades à Lourdes, tiré de la thèse de l'auteure, a obtenu l'année de sa parution (2006), le prix franco-japonais Shibusawa-Claudel. Boursière du gouvernement japonais en France de 1991 à 1995, l'auteure a soutenu en 1993 un DEA d'Histoire et Civilisations à l'École des hautes études en sciences sociales, intitulé : « La place des malades au sanctuaire de Lourdes : 1874-1977 » dirigé par André Godin et Philippe Boutry. Ce mémoire annonçait déjà les prémisses de l'ouvrage ici recensé. Basé sur un travail minutieux de collecte des matériaux (archives officielles, publications locales, sources audiovisuelles), il s'appuie également sur de solides données sociologiques (nombreuses statistiques) et sur des terrains ethnographiques réguliers qui se sont déroulés de 1992 à 1999.

2 L'étude commence par une substantielle introduction qui traite, successivement, du "monde de Lourdes", de Lourdes en chiffres et des recherches sur Lourdes. L'introduction dévoile également les intentions de l'auteure. Comme l'indique le titre de l'ouvrage, le parti pris est de mettre les malades au centre de l'étude qui s'appuie, globalement, sur la lecture socio-anthropologique des pèlerinages de Victor Turner et sur l'interactionisme développé par le sociologue Ervin Goffman. À la différence d'autres études pèlerines, ne sont guère traités dans cet ouvrage les pèlerins isolés ou les simples touristes, mais principalement les pèlerins organisés et surtout les associations qui les entourent et les soutiennent aux deux sens moral et financier. Cette approche du pèlerinage de Lourdes constitue, tout à la fois, la nouveauté de l'ouvrage mais aussi peut-être ses limites, si l'on songe au lectorat concerné. Dans le cas précis, un public japonais qui ne dispose pas d'étude générale sur le pèlerinage. Cette contribution aux recherches sur Lourdes part de deux interrogations fortes : pourquoi des malades et des handicapés à Lourdes? Pourquoi tant de bénévoles pour les aider? 
Le regard comparé qu'offre l'étude de Nathalie Kouamé sur le pèlerinage de Shikoku (Pèlerinage et société dans le Japon des Tokugawa. Le pèlerinage de Shikoku entre 1598 et 1868. Paris, École française d'Extrême-Orient, 2001; Arch. 120-23, 2002), où les pratiques japonaises d'hospitalité et d'accueil (coutume du settai) sont finement analysées, est à cet égard instructif quant aux attentes différenciées des pèlerins chrétiens et bouddhistes.

3 La première partie du livre traite des particularités et des transformations de l'espace pèlerin qui se décline en plusieurs lieux : le domaine de la grotte et les alentours, la basilique, l'esplanade et la prairie, le domaine sud du sanctuaire et la ville de Lourdes. Se basant sur l'analyse de la composition de l'espace, de son décor et des rituels qui y sont pratiqués, l'auteure montre comment la place des malades a évolué dans le temps, passant, pour les uns, d'un espace spectaculaire à un espace intime; pour les autres, d'un espace collectif à un espace privé.

4 La deuxième partie est consacrée à l'histoire du pèlerinage de Lourdes vue comme l'établissement progressif d'un "pèlerinage de malades", depuis les premiers pèlerinages paroissiens des années 1866-1868 où le thème de la guérison est déjà présent, jusqu'à l'internationalisation du pèlerinage à partir de l'après-guerre qui se décline autour des thèmes de la réconciliation et de la paix. J. Terado analyse de manière détaillée, en s'appuyant sur les archives diocésaines, la « résurrection des paroisses ", les premiers récits de guérison, le «pèlerinage national» qui devient "pèlerinage national des malades». S'inspirant des analyses de Susan Sontag sur la maladie comme métaphore, elle montre également que la douleur peut aussi symboliser la guérison attendue d'une France malade (de sa défaite contre l'Allemagne), à l'exemple du "pèlerinage des bannières" de 1872. Puis, progressivement, Lourdes n'est plus seulement "notre pèlerinage »; il déborde le cadre national pour devenir le rassemblement mondial de l'ensemble des catholiques.

5 La troisième partie est une analyse très précise des associations de bénévoles dédiées aux pèlerins, et tout spécialement aux malades et aux handicapés: les Hospitalités. Après un exposé sur les transformations sociales qu'ont connues ces confréries de laïcs, œuvres secourables et catholicisme social sont introduits par le biais des valeurs chrétiennes qu'ils véhiculent : charité et philanthropie. Deux associations reçoivent un traitement à la hauteur de leur histoire : l'Hospitalité Notre-Dame de Salut (1880) et l'Hospitalité Notre-Dame de Lourdes (1882). Fondation, règles, types d'activités, mais aussi composition de leurs membres et « esprit » (seishin) - ou pour employer un terme à connotation religieuse, «spiritualité hospitalière» - qui les animent, sont successivement présentés avec force détails. L'auteure met l'accent sur la signification d'une " charité masculine » dans le cas de la première Hospitalité en faisant référence à sa fondation aristocratique qu'elle caractérise par l'expression "noblesse oblige", même si les hommes appartenant aux classes supérieures n'étaient guère nombreux par rapport à l'ensemble des hospitaliers. La description et l'analyse de ces deux associations historiques montrent également que les diocèses qui, dans un premier temps, participaient au Pèlerinage National, ont créé eux aussi, peu à peu, leur propre pèlerinage. Entre l'Hospitalité de Notre-Dame de Lourdes et les diocèses, un réseau s'est constitué à l'intérieur duquel le «monde du pèlerinage de Lourdes» n'est plus circonscrit au seul territoire de la ville.

6 La quatrième partie, intitulée "Les guérisons miraculeuses ", et la cinquième, "Le développement du pèlerinage des malades ", recentrent le propos sur les thèmes 
majeurs de l'ouvrage : grâce divine, maladie et guérison. S'appuyant sur les archives du Bureau des contestations médicales, la place, le rôle et l'influence des médecins, ainsi que le processus de "la médicalisation » de la société française contemporaine, sont mesurés à l'aune du sens que revêt pour les participants ce "pèlerinage des malades ». Les guérisons miraculeuses sont ainsi analysées à travers un riche corpus de sources extrêmement diverses réparties selon deux catégories: les preuves médicales et les récits des pèlerins. La réflexion vise, pour reprendre les propres termes de l'auteure, à répondre à la question suivante: "que devaient être les guérisons miraculeuses de Lourdes? », ou, selon une autre formulation: " qu'est-ce qu'une guérison miraculeuse "appropriée" (tekisetsuna) ?». L'analyse introduit d'intéressantes distinctions entre, par exemple, le récit à la première personne de celui ou celle qui raconte sa guérison et les premiers témoignages souvent produits par des tiers (la famille, le corps médical etc.) L'auteure montre avec acuité les changements de modalités des discours tant sur les malades que sur les guérisons miraculeuses. La méthode de l'observation participante livre à ce moment d'utiles commentaires à l'analyse qui se déploie alors dans le temps présent. L'histoire des divers types de pèlerinages, à partir d'une réflexion centrée sur la place accordée aux malades ou revendiquée par ces derniers, rend compte des transformations opérées au cours $\mathrm{du} \mathrm{xx}^{\mathrm{e}}$ siècle : évolutions des mentalités pèlerines mais aussi des rituels en tant qu'actions envers soi ou envers les autres.

7 Par-delà la présentation succincte de L'univers du pèlerinage des malades à Lourdes, il reste à dire la richesse d'une étude qui, par des incises bienvenues, des angles d'attaque diversifiés, offre nombre de questionnements et de réflexions originaux sur les transformations $\mathrm{du}$ catholicisme français; sur la tension entre le politique et le religieux; sur la différenciation entre l'individuel et le collectif; sur le «nous » et les " autres »; sur la maladie, la vieillesse et la mort; sur le particulier et l'universel. Ces considérations confèrent à l'ouvrage une ampleur qui va bien au-delà du seul pèlerinage des malades et lèvent, à la fin de la lecture, les doutes du début sur la pertinence des choix de J. Terado. En cette année du $150^{\mathrm{e}}$ anniversaire de l'apparition de la Vierge à Lourdes, saluons cet ouvrage japonais original et très complet, et formulons le vœu qu'un petit miracle de l'édition puisse le faire connaitre aux lecteurs occidentaux. Faisant suite à l'ouvrage de l'ethnologue Kazutoshi Seki sur les apparitions de la Vierge (Les apparitions de la Vierge. Réflexions sur le catholicisme populaire moderne, Seibo no shutsugen. Kindai Fôku katorisizumu-kô, Tôkyô, Nihon Edeitâ, 1993), cette publication est un nouveau maillon d'une socio-anthropologie des pratiques et croyances mariales françaises présentée par nos collègues japonais. Comme souvent, l'édition est digne d'éloges: photos, notes de bas de page, larges marges autour du texte, termes techniques indiqués en français, sources documentaires, bibliographie de plusieurs dizaines de pages et index, sont les bienvenus. 